\title{
BDA heralds breakthrough on Dental Assurance Review
}

The BDA has heralded a breakthrough in talks with the NHS Business

Services Authority to provide a fair and proportionate approach to new Dental Assurance Reviews (DARs).

The BSA announced last year that they were going to commence four new reviews from September 2019 looking at claims for band 3 inlays, incomplete treatment (band 2 and/or 3), band 2 fissure sealants and band 1 urgent claims followed by a band 2 with no examination.

The BSA had also published a series of 'Spotlight' articles advising dentists on correct ways of claiming for work done. The BDA believes that some of these articles apply a level of interpretation to what is an appropriate claim far beyond what is set out in regulations. In some instances, they also stray into advice on clinical practice that is not the BSA's remit.

The BDA had expressed grave concerns that the previous 28 -day re-attendance reviews were bringing in an ever-greater number of practices for scrutiny, rather than targeting genuine outliers, and in doing so deterring legitimate claims. In response to BDA challenges to justify its selection process the BSA has now reviewed its approaches, and confirmed a move to focus scrutiny on a very small number of practices that are genuinely extreme outliers, amounting to $0.2-0.8 \%$ of providers, around 100 in total.

Contracts now identified for the DAR will receive a letter informing them of their outlier status, asking them to consider this and an offer from the BSA to provide additional information. Contractors will have six months to consider their claiming behaviour and implement any changes necessary.

Only a tiny number of practices showing no or very little reduction in extreme outlier status will be required to complete a self-audit. Where contractors fail to comply with conducting a self-audit or do not satisfactorily conduct one, the contract will then be subject to a clinical record review of a small number of claims by a clinical adviser. The outcome of this would be provided to the contractor. If agreement is then reached, the contract would then likely be moved out of the DAR.

Where no agreement can be reached on the clinical record review then a full record review will be conducted on all claims identified for the self-audit and this would likely lead to a referral to the local NHS area team. The BSA now intend to give providers every opportunity to avoid this.

Chair of the BDA's General Dental Practice Committee Dave Cottam said: 'BDA members had expressed profound concerns over these reviews. We called for a halt, and the BSA to their credit have listened.

'We now have review criteria at an acceptable level, meaning only a fraction of $1 \%$ of providers deemed extreme outliers will need to participate. Instead of heaping unacceptable burdens on a growing number of practices, we have a proportionate approach.

'The Spotlight series are still under review, and we will continue to press the BSA to modify their advice. We have secured a significant improvement in the DAR process that should mean that the scrutiny applied is targeted and reasonable. The net result is no practice should face a grilling for entirely appropriate claiming.'

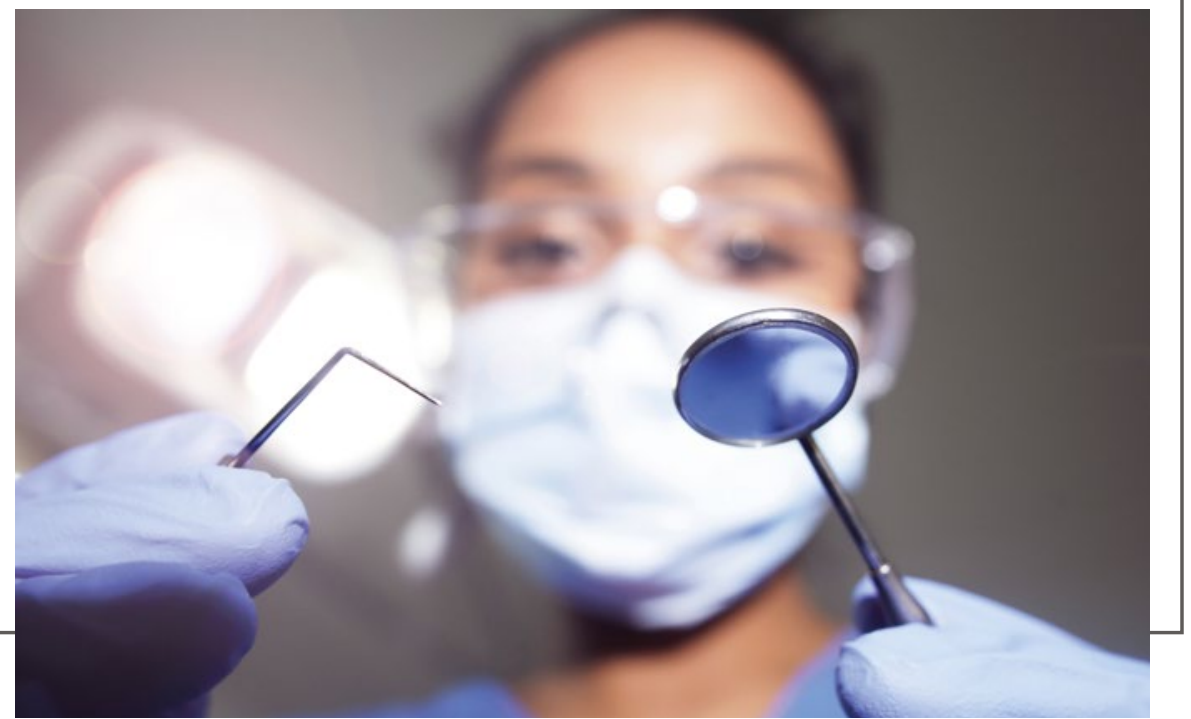

